\title{
Minimum Variance Signal Selection for Aorta Radius Estimation Using Radar
}

\author{
Lars Erik Solberg,, ${ }^{1}$ Svein-Erik Hamran,, ${ }^{2,3}$ Tor Berger, ${ }^{2}$ and Ilangko Balasingham 1,4 \\ ${ }^{1}$ Interventional Centre, Oslo University Hospital and Interventional Centre, Institute of Clinical Medicine, \\ University of Oslo, Sognsvannsveien 20, 0027 Oslo, Norway \\ ${ }^{2}$ Forsvarets forskningsinstitutt, Postboks 25, 2027 Kjeller, Norway \\ ${ }^{3}$ Department of Geosciences, University of Oslo, P.O. Box 1047 Blindern, 0316 Oslo, Norway \\ ${ }^{4}$ Department of Electronics and Telecommunications, Norwegian University of Science and Technology (NTNU), \\ 7491 Trondheim, Norway
}

Correspondence should be addressed to Lars Erik Solberg, lars.erik.solberg@gmail.com

Received 9 March 2010; Accepted 7 June 2010

Academic Editor: Christophoros Nikou

Copyright ( 2010 Lars Erik Solberg et al. This is an open access article distributed under the Creative Commons Attribution License, which permits unrestricted use, distribution, and reproduction in any medium, provided the original work is properly cited.

This paper studies the optimum signal choice for the estimation of the aortic blood pressure via aorta radius, using a monostatic radar configuration. The method involves developing the Cramér-Rao lower bound (CRLB) for a simplified model. The CRLB for model parameters are compared with simulation results using a grid-based approach for estimation. The CRLBs are within the 99\% confidence intervals for all chosen parameter values. The CRLBs show an optimal region within an ellipsoid centered at $1 \mathrm{GHz}$ center frequency and $1.25 \mathrm{GHz}$ bandwidth with axes of $0.5 \mathrm{GHz}$ and $1 \mathrm{GHz}$, respectively. Calculations show that emitted signal energy to received noise spectral density should exceed $10^{12}$ for a precision of approximately $0.1 \mathrm{~mm}$ for a large range of model parameters. This implies a minimum average power of $0.4 \mu \mathrm{W}$. These values are based on optimistic assumptions. Reflections, improved propagation model, true receiver noise, and parameter ranges should be considered in a practical implementation.

\section{Introduction}

Our research effort addresses the issue of estimating the central blood pressure by observing the radius of the aorta as a function of time using radar techniques and thereby establishing a noninvasive technique.

Noninvasive measurements of blood pressure (BP) can be performed using the sphygmomanometer, photoplethysmograph [1], tonography [2], and pulse transit time [3]. However, they all rely on peripheral measurement points. This may constitute a problem in certain situations such as when flow redistribution to central parts of the body (heavy injury, temperature, etc.) degrades these measurements; another situation where central measurements may prove advantageous is in presence of strong movements of the peripheral locations which affect pressure measurements [4].

The use of radar-based approaches in a medical context is neither new nor common. An interesting overview of the use of radar for medical applications is presented in [5], which traces research back to the late 1970s. It seems that renewed interest has been spurred following McEwan's Micropower Impulse Radar [6] in the early 1990s which combined ultrawide band (UWB) pulses with very low power, small size, and low system cost. It also seems that some of this momentum in research related to UWB pulses has been founded on dubious claims of exceptional behavior related to the impulsive nature of the signal, such as specific penetration, resonances, and presumed inadequacy of a Fourier type description, which have been refuted [7].

The research into medical sensor applications include apexcardiography, heart rate, respiration rate, heart-rate variability, blood pressure pulse transit time based on peripheral locations, and associated applications such as through rubble or walls vital signs detection $[5,6,8]$. With respect to imaging, the use of an antenna array for the early detection of breast cancer $[9,10]$ should be mentioned. 
The present research activities on breast cancer and vital signs detections differ with respect to our objective of estimating blood pressure. In breast cancer detection, the concerned tissues are predominantly less lossy whereas in respiration and heart-rate estimation the radar signature can be due to the air-skin interface [11]. Finally, our active use of a cylindrical target structure distinguishes our approach from those mentioned.

1.1. Physiological Problem Description. The aim of our project is the estimation of blood pressure and possibly other clinically pertinent parameters. We believe the following phenomena apply to the aorta and, hence, may serve as the basis for estimation approaches.

(1) Sugawara et al. [12] showed a linear relationship between percentage changes in instantaneous blood pressure and diameter, based on measurements on the carotid artery.

(2) According to $[3,4,13]$, there is a nonlinear relationship between mean arterial pressure $(\bar{P})$ and compliance (see below).

Common to the above mentioned approaches, the radarbased method will need to estimate the aortic diameter as a function of time $(d(t))$.

The key point of the approaches based on the second phenomena is the relationship between the elasticity, of which compliance is a measure, of a homogeneous, circular tube and the speed of propagation of a pressure pulse along the tube and presented by Otto Frank in 1926 (according to [14]),

$$
v=\sqrt{\frac{K_{L}}{\rho}}=\sqrt{\frac{1}{\rho C_{L}}}, \quad C_{L}=\frac{d A}{d P}=\frac{1}{K_{L}},
$$

where $v$ is the speed of the pulse propagating along the aorta, $K_{L}$ is the bulk elastic modulus per unit length, $C_{L}$ is the compliance, $A$ is cross-sectional area, $P$ is arterial pressure, and $\rho$ is the blood density $\left(\rho\right.$ is $\left.1.05 \mathrm{~g} / \mathrm{cm}^{3}\right)$. Compliance is used by clinicians as a local measure of arterial elasticity. This equation directly relates pulse velocity to compliance. An often-cited and similar formulation of this relationship is provided in Moen-Korteweg's equation which uses the incremental Young's elastic modulus $E_{\text {inc }}$,

$$
v=\sqrt{\frac{E_{\text {inch }}}{\rho(2 r)}}=\sqrt{\frac{1}{2 \rho} \frac{\Delta P}{\Delta r / r}}, \quad E_{\text {inc }}=\frac{\Delta P}{\Delta r / r} \cdot \frac{r}{h},
$$

where $\Delta r$ is a change in aortic radius associated with a change in pressure $\Delta P$ at an aortic radius $r$, and $h$ is the aortic wall thickness. Hence, the parameters $(v, r, \Delta r)$ provide sufficient information for estimating $C_{L}$ and thereby $\bar{P}$ based on the above nonlinear relationship. As a by-product, the procedure also provides for heart rate (HR), and possibly an indication on pulse pressure.

The diameter variations of the aorta have been measured by Stefanidis [15] using a precise and invasive measurement method based on pressure and diameter sensors introduced through catheters. It concludes that typical diameter peakto-peak amplitudes for a normal population is $2.18 \pm 0.44 \mathrm{~mm}$. This means the measurement precision of the aorta diameter variations must be at a fraction of a millimeter, a strict requirement also for a radar-based method.

1.2. Object of the Current Article. In anticipation of the expected strong attenuation in our application, the current article addresses issues related to the obtainable precision from a system's point of view. What criterion may be identified in order to achieve the required performance? To answer this question, the Cramér-Rao lower bound (CRLB) is used as a selection criterion, and which will map the performance for a range of parameter values. System parameters of interest include the necessary energy/power and optimum choices for center frequency and bandwidth, if such optima exist. In this approach, we will focus on the properties of the human body as a channel thereby disregarding the antenna selection. This implies that in the joint antenna and channel system, we are only optimising the second subsystem and tacitly assuming that an appropriate antenna exists.

The medium in which the radar signal propagates is lossy and dispersive, and the geometry is complex, see Figure 1. To answer the above question a simulations-based approach could be considered, however, it would be slow and may not provide further insight into the problem. Instead, we have opted for an analytical approach based on a mathematical representation of the channel and on the derivation of the CRLB. In order to obtain a mathematically tractable model, a simplified geometry is used: we consider a $2 \mathrm{D}$ problem with a cylinder of time-varying radius of lossy material immersed into a region of a different lossy material. Between the transmitter and aorta and between the aorta and receiver antenna the propagation model is planar. The time variation is considered to be static at each measurement instant, while dynamic between measurements. The estimation problem is that of estimating the radius of the cylinder without knowledge of its depth, and by allowing the subtraction of two responses separated in time and corresponding to distinct radii. Justification of this model simplification will be elaborated in subsequent sections.

This choice of geometry departs from a realistic scenario especially by disregarding multipath components reflected via the aorta. It also assumes the aorta is the only dynamic tissue with a significant response within the relevant range depth. This hypothesis may prove wrong as several organs in the human body, for instance, the lungs and the stomach, are in motion and may be a source of clutter within the relevant range. Also disregarded, reflection and transmission coefficients at tissue boundaries may lead to increased path loss. These effects will probably degrade estimator precision. Therefore, the results obtained in this paper, by limiting its scope to a simplified geometry, may prove optimistic in a realistic scenario.

The above problem statement is akin to the estimation of range in a classical radar context, to delay in communications or to localization in wireless networks, where the CRLB 


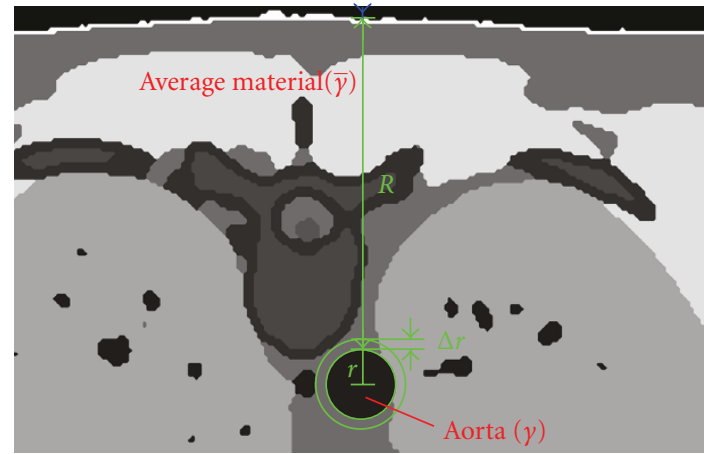

FIGURE 1: The image represents a gray scale encoding of tissue types and includes cancellous bone, bone marrow, blood, lungs, muscle, fat, skin, nerves, and so forth. In the model, all but the blood contained in the aorta contribute to an average material $(\bar{\gamma})$ based on surface areas. The two circles around the aorta represent the aorta at two different instants; $r, R$ refers to the first of these, while $r+\Delta r, R-\Delta r$ would refer to the second.

and the maximum likelihood estimation are well defined. However, due to the lossy channel, these results cannot be applied directly. To the best of the authors' knowledge, the CRLB for a comparable problem has not been established; most results assume channels with signals propagating essentially in nondispersive, nonlossy materials and focus on channel behavior in statistical terms and in which multiple paths exist between transmitter and receiver. Another common objective for the development of CRLBs has been in analyzing performance of modulation techniques. The survey in [16] provides an overview of lower bounds in timedelay estimation.

After a brief presentation of mathematical notation in Section 2, in Section 3, we will derive an analytic expression for the CRLB for a general channel model, yet will evaluate this expression numerically for our specific channel because even the most simplified model would result in integrals without closed forms. The numerical results show that there exists an optimum choice of center frequency $\left(f_{c}\right)$ and bandwidth $(B)$ when ranges of parameter values are considered. In Section 4, the theoretic results are simulated for a set of system and parameter values $\left\{\left(f_{c}, B, R, r, \Delta r\right)_{n}\right\}$ which will show a tight correspondence between theory and simulation. These results are discussed in Section 5, where also system performance in terms of target precision will be discussed. Section 6 concludes on the findings in this paper.

\section{Mathematical Notation}

In the expressions that follow, lower-case letters refer to signals in the time domain-normal if continuous $(x(t), s(t), n(t))$ and bold-face if sampled (vector format; $\mathbf{x}[m], \mathbf{s}[m], \mathbf{z}[m])$; depending on context, these vectors may represent random variables. Upper-case letters refer to the frequency domain-calligraphic style if random variables $(\mathcal{X}, \mathcal{N})$, else bold-faced for vectors and matrices $(\mathbf{K}, \mathbf{X}, \mathbf{Z}, \mathbf{S})$, while normal-faced for continuous variables $(S, X)$. $\boldsymbol{\theta}$ denotes the true parameter values in a space $\Theta$ of dimension $p, \hat{\boldsymbol{\theta}}_{\mathrm{ML}}$ is the Maximum Likelihood (ML) estimate, and $\hat{\boldsymbol{\theta}}$ is some estimate of $\boldsymbol{\theta}$. Eventually, the model will include three parameters: $\boldsymbol{\theta}=[R, r, \Delta r]^{T}$.

Subscripts will be used to signify that the associated variable is parametrized (e.g., $\mathbf{H}_{\boldsymbol{\theta}}, \mathbf{M}_{\boldsymbol{\theta}}$ ).

The contents of a matrice (e.g., $A$ ) is written $A=\left[a_{i j}\right]$, where $i$ denotes row indices and $j$ denotes column indices. If $\mathbf{Z}_{\boldsymbol{\theta}}$ is a vector parametrized by a vector $\boldsymbol{\theta}$, then its derivative with respect to $\boldsymbol{\theta}$ is defined as

$$
\mathbf{Z}_{\boldsymbol{\theta}}^{\prime}=\frac{d \mathbf{Z}_{\boldsymbol{\theta}}}{d \boldsymbol{\theta}}=\left[\frac{d \mathbf{Z}_{\theta}[i]}{d \boldsymbol{\theta}_{j}}\right]
$$

In the interest of concise notation, the following innerproduct in the Hilbert Space of finite (length $N$ ) complex sequences will be used:

$$
\begin{gathered}
\mathbf{b}, \mathbf{a} \in \mathbb{C}^{N}, \quad \mathbf{K}^{-1}=\left(\mathbf{K}^{-1}\right)^{H} \in \mathbb{C}^{N \times N}, \\
\langle\mathbf{a}, \mathbf{b}\rangle=\mathbf{b}^{H} \mathbf{K}^{-1} \mathbf{a}, \quad\|\mathbf{a}\|^{2}=\langle\mathbf{a}, \mathbf{a}\rangle, \\
\frac{d}{d \boldsymbol{\theta}_{i}}\left\langle\mathbf{a}_{\boldsymbol{\theta}}, \mathbf{b}_{\boldsymbol{\theta}}\right\rangle=\left\langle\frac{d \mathbf{a}_{\boldsymbol{\theta}}}{d \boldsymbol{\theta}_{\boldsymbol{i}}}, \mathbf{b}_{\boldsymbol{\theta}}\right\rangle+\left\langle\mathbf{a}_{\boldsymbol{\theta}}, \frac{d \mathbf{b}_{\boldsymbol{\theta}}}{d \boldsymbol{\theta}_{i}}\right\rangle .
\end{gathered}
$$

Here, a subscript $t$ will be added when $\mathbf{K}=\mathbf{K}_{n, t}$, otherwise $\mathbf{K}=\mathbf{K}_{n, f}$ will be assumed; these matrices will be defined shortly.

For mathematical simplicity, instead of using the standard DFT, we will assume the unitary equivalent $\left(\mathrm{DFT}_{U}\right)$ :

$$
\begin{aligned}
\mathbf{A}[k] & =\frac{1}{\sqrt{N}} \sum_{m=0}^{N-1} \mathbf{a}[m] e^{-j 2 \pi m k / N}, \\
\langle\mathbf{a}, \mathbf{b}\rangle_{t} & =\langle\mathbf{A}, \mathbf{B}\rangle, \quad \text { where } \mathbf{a} \stackrel{\mathrm{DFT}_{U}}{\longrightarrow} \mathbf{A} \wedge \mathbf{b} \stackrel{\mathrm{DFT}_{U}}{\longleftrightarrow} \mathbf{B} .
\end{aligned}
$$

As a unitary operator is defined by the condition that the adjoint of the transform is its own inverse, it conserves the inner product (5), and therefore also the norm.

\section{CRLB}

Several lower bounds have been developed to describe estimator's precision of which the CRLB and Ziv-Zakai lower bound (ZZLB) are currently the most frequently employed. The latter has been specifically developed for delay estimation in the objective of improving the accuracy of the bound at low SNR when ambiguous peaks tend to decrease the obtainable precision over the CRLB and $a$ priori knowledge limits the variance of the estimator. In our context, the necessary accuracy of estimation is expected to require a sufficient SNR for the receiver performance to exceed the threshold at which the ZZLB provides for a more accurate lower bound. Incidentally, studies have shown that the threshold effect may be pushed towards lower SNR if some prior information may constrain the estimates to vary around the true maximum likelihood peak [17]. We have therefore focused on the CRLB. 
3.1. General Transfer Function $\mathbf{H}_{\boldsymbol{\theta}}$. In a first stage, we will consider the following generic signal model. A signal $(s)$ is emitted by the transmitter and passes through a generic channel $\left(\mathbf{H}_{\boldsymbol{\theta}}, h_{\boldsymbol{\theta}}\right)$, which depends on a set of parameters $(\boldsymbol{\theta})$, and is corrupted by an uncorrelated, wide-sense stationary (WSS) random Gaussian process $(n)$ bandlimited to $W$ Hertz and independent of the model parameters. The signal plus noise is then observed $(x)$ as follows:

$$
\begin{aligned}
x(t) & =\left\{h_{\boldsymbol{\theta}} \star s\right\}(t)+n(t)=z_{\boldsymbol{\theta}}(t)+n(t), \\
R_{n}(\tau) & \triangleq E[n(t) \bar{n}(t-\tau)] \stackrel{F T}{\longleftrightarrow} \Gamma_{n}(f),
\end{aligned}
$$

where $R_{n}$ is the noise autocorrelation and $\Gamma_{n}$ its power spectral density (PSD).

In order to develop the CRLB, a stochastic model of the above in the form of a probability distribution is needed. Then expressions for the score and subsequently the Fisher Information Matrix (FIM) are derived, after which a channel model will be discussed.

As shown in [18, Chapter 2], the information in a bandlimited random process observed over a time interval $T$ is uniquely represented by values of samples spaced $\Delta t=1 /(2 W)$ apart by virtue of the Nyquist-Shannon sampling theorem: any and every realization of the process is represented by its sample values at these intervals because the realizations may be recreated by interpolating with the ideal interpolating function (a sinc for signals of infinite duration). The distribution of the sampled, stationary, random Gaussian process is [19]

$f_{\theta}(\mathbf{x})=c_{n} \operatorname{Exp}\left\{-\frac{1}{2}\left\|\mathbf{x}-\mathbf{z}_{\theta}\right\|_{t}^{2}\right\}, \quad \mathbf{K}_{n, t}=\left[R_{n}\left(t_{j}-t_{i}\right)_{i j}\right]$,

where $\mathbf{x}, \mathbf{z}_{\boldsymbol{\theta}}$ are the sample vectors of length $N=2 M+1, c_{n}$ is a normalizing constant independent of $\boldsymbol{\theta}$, and $\mathbf{K}_{n, t}$ is the noise covariance matrix in the time domain.

For sufficient observation time $T$, the discrete Fourier transform (DFT) coefficients are essentially independent random variables as are the real and imaginary parts. In the development by Van Trees [19, Volume 1, Chapter 3], it was shown that transform coefficients are uncorrelated when the orthonormal basis is composed of eigenvectors of the covariance of the random process. Large observation time means the eigenvectors tend towards complex exponentials.

Under these conditions, the distribution in the frequency domain can be shown as

$$
\begin{aligned}
f_{\theta}(\mathcal{X}) & =c_{n} \operatorname{Exp}\left\{-\frac{1}{2}\left\|\mathcal{X}-\mathbf{Z}_{\theta}\right\|^{2}\right\} \\
\mathbf{K}_{n, f} & =E[\mathcal{N}[i] \overline{\mathcal{N}[j]}]=\operatorname{diag}\left(\frac{\Gamma_{n}\left(f_{k}\right)}{\Delta t}\right),
\end{aligned}
$$

where $\mathbf{K}_{n, f}$ is the covariance matrix in the frequency domain.
We see that both the time-domain and frequencydomain distributions show that the maximum-likelihood estimator is also the nonlinear least-squares solution:

$$
\text { Frequency domain }: \begin{aligned}
\boldsymbol{\theta}_{\mathrm{ML}}= & \underset{\boldsymbol{\theta} \in \boldsymbol{\Theta}}{\operatorname{Argmin}}\left\{\left\|\mathcal{X}-\mathbf{Z}_{\boldsymbol{\theta}}\right\|^{2}\right\} \\
= & \underset{\boldsymbol{\theta} \in \boldsymbol{\Theta}}{\operatorname{Argmin}}\left\{\left\|\mathbf{Z}_{\boldsymbol{\theta}}\right\|^{2},\right. \\
& \left.-2 \operatorname{Re}\left(\left\langle\mathcal{X}-\mathbf{Z}_{\boldsymbol{\theta}}\right\rangle\right)\right\},
\end{aligned}
$$

$$
\text { Time domain }:=\underset{\boldsymbol{\theta} \in \boldsymbol{\Theta}}{\operatorname{Argmin}}\left\{\left\|\mathbf{x}-\mathbf{z}_{\boldsymbol{\theta}}\right\|_{t}^{2}\right\} \text {. }
$$

In the case where the signal channel simply introduces a delay, $\left\|\mathbf{Z}_{\boldsymbol{\theta}}\right\|^{2}$ is independent of $\boldsymbol{\theta}$ and the second term in (9) should hence be maximized. By using the CauchySchwartz inequality, this optimization can be shown to be identical to searching for the maximum of the crosscorrelation. However, here both the norm and signal form $\left(\mathbf{z}_{\boldsymbol{\theta}}\right)$ are dependent upon $\boldsymbol{\theta}$ and hence the "matched filter" corresponds to a search over the parameter space $(\Theta)$ of dimension $p$.

The score is the derivative of the log-likelihood,

$$
\begin{aligned}
s(\boldsymbol{\theta} ; \mathcal{X}) & =\frac{d}{d \boldsymbol{\theta}} \ln \left(f_{\theta}(\mathcal{X})\right) \\
& =-\frac{1}{2} \frac{d}{d \boldsymbol{\theta}}\left\|\mathcal{X}-\mathbf{Z}_{\boldsymbol{\theta}}\right\|^{2} \\
& =\left[\operatorname{Re}\left(\left\langle\mathcal{X}-\mathbf{Z}_{\boldsymbol{\theta}}, \frac{d \mathbf{Z}_{\boldsymbol{\theta}}}{d \boldsymbol{\theta}_{i}}\right\rangle\right)\right] \in \mathbb{R}^{p} .
\end{aligned}
$$

In the theory of maximum likelihood estimators, $\hat{\boldsymbol{\theta}}_{M L}$ is chosen such that score becomes null.

Next, the FIM $(\mathbf{J}(\boldsymbol{\theta}))$ is defined either through the variance of the score, which has expectation zero, or equivalently through the expected value of the double-derivative,

$$
\begin{aligned}
\mathbf{J}(\boldsymbol{\theta})_{i j} & =-E\left[\frac{d}{d \boldsymbol{\theta}_{j}} s(\boldsymbol{\theta} ; \mathcal{X})_{i}\right] \\
& =-E\left[\operatorname{Re}\left(-\left\langle\frac{d \mathbf{Z}_{\boldsymbol{\theta}}}{d \boldsymbol{\theta}_{j}}, \frac{d \mathbf{Z}_{\boldsymbol{\theta}}}{d \boldsymbol{\theta}_{i}}\right\rangle+\left\langle\chi-\mathbf{Z}_{\boldsymbol{\theta}}, \frac{d^{2} \mathbf{Z}_{\boldsymbol{\theta}}}{d \boldsymbol{\theta}_{i} d \boldsymbol{\theta}_{j}}\right\rangle\right)\right] \\
& =\operatorname{Re}\left(\left\langle\frac{d \mathbf{Z}_{\boldsymbol{\theta}}}{d \boldsymbol{\theta}_{j}}, \frac{d \mathbf{Z}_{\boldsymbol{\theta}}}{d \boldsymbol{\theta}_{i}}\right\rangle\right), \\
\mathbf{J}(\boldsymbol{\theta}) & =\operatorname{Re}\left(\frac{d \mathbf{Z}_{\boldsymbol{\theta}}{ }^{H}}{d \boldsymbol{\theta}} \mathbf{K}_{n, f}^{-1} \frac{d \mathbf{Z}_{\boldsymbol{\theta}}}{d \boldsymbol{\theta}}\right) .
\end{aligned}
$$

Equation (12) uses the fact that the expectation $E[X]$ is $\mathbf{Z}_{\boldsymbol{\theta}}$. Although each element may be formulated as an inner product, $\mathbf{J}(\boldsymbol{\theta})$ may not be formulated as an inner product of matrices $\mathbf{Z}_{\boldsymbol{\theta}}^{\prime}$. Using the model $\mathbf{Z}_{\boldsymbol{\theta}}[k]=\mathbf{H}_{\boldsymbol{\theta}}[k] \mathbf{S}[k]$ we get

$$
\mathbf{J}(\boldsymbol{\theta})=\sum_{k=-M}^{M} \frac{|\mathbf{S}[k]|^{2}}{\mathbf{K}_{n, f}[k, k]} \operatorname{Re}\left(\frac{d \mathbf{H}_{\boldsymbol{\theta}}[k]}{d \boldsymbol{\theta}} \frac{d \mathbf{H}_{\boldsymbol{\theta}}[k]}{d \boldsymbol{\theta}}\right) .
$$


Using the approximations $S\left(f_{k}\right) \approx \Delta t \sqrt{N} \mathbf{S}[k], \mathbf{K}_{n, f}[k, k] \approx$ $\Gamma_{n}\left(f_{k}\right) / \Delta t$, and $1 / T=d f$, and assuming sufficient observation time,

$$
\mathbf{J}(\boldsymbol{\theta})_{i j} \approx \int_{W} \frac{|S(f)|^{2}}{\Gamma_{n}(f)} \operatorname{Re}\left(\frac{d H_{\theta}(f)}{d \boldsymbol{\theta}_{i}} \frac{\overline{d H_{\boldsymbol{\theta}}(f)}}{d \boldsymbol{\theta}_{j}}\right) d f
$$

The CRLB are the values along the diagonal of the inverse of the FIM, $\sigma_{\theta_{i}}^{2} \geq \mathbf{J}(\boldsymbol{\theta})_{i i}^{-1}$. It follows that, generally, the lowest CRLB for an estimator is achieved when the FIM is maximum. Also, the CRLB of different parameters are mutually related through the inversion of the matrix.

Given that both $\Gamma_{n}$ and $\mathbf{H}_{\boldsymbol{\theta}}$ are determined by the measurement situation, what remains is an intelligent choice of the signal in order to enhance those frequencies such that the lower bound becomes minimum. For simplicity, assuming the signal is an ideal bandpass signal and the noise is white $\left(\Gamma_{n}(f)=\Gamma_{0}\right)$, the above may be reformulated as

$$
\begin{aligned}
|S(f)|^{2} & =\frac{E_{s}}{2 B}\left\{\operatorname{rect}\left(\frac{f-f_{c}}{B}\right)+\operatorname{rect}\left(\frac{f+f_{c}}{B}\right)\right\}, \\
\mathbf{J}(\boldsymbol{\theta})_{i j} & \approx \frac{E_{s}}{\Gamma_{0}} \frac{1}{B} \int_{f_{c}-B / 2}^{f_{c}+B / 2} \operatorname{Re}\left(\frac{d H_{\boldsymbol{\theta}}(f)}{d \boldsymbol{\theta}_{i}} \frac{\overline{d H_{\boldsymbol{\theta}}(f)}}{d \boldsymbol{\theta}_{j}}\right) d f,
\end{aligned}
$$

where $E_{s}$ is the energy of the continuous signal and $\Gamma_{0}$ the white noise spectral density, while $B$ is the signal bandwidth and $f_{c}$ its center frequency. This shows that the CRLB scales linearly with the ratio $\Gamma_{0} / E_{s}$. This is the reciprocal of the transmitted signal energy $\left(E_{s}\right)$ to the noise spectral density $\left(\Gamma_{0}\right)$. If the signal energy had been referred to the receiver end, this would have been the signal-to-noise ratio. This expression (15) shows that the FIM components are maximum when the average value of the integrand is maximum. More generally, the signal PSD effectively weighs the channel components.

3.2. Channel Model. A key objective with a mathematical model as opposed to complex simulations is, in addition to less computational burden, the facility of analyzing the influence of different system parameters. However, primarily due to the above integral even the simplest of models, for instance, a layered representation, fails to allow for a closedform solution because the material properties complicate the issue.

In Figure 1, a gray scale encoding of tissues is presented based on the Voxel Man [20] data set, which has in turn been based on the Visible Human Project [21]. This figure shows that the channel between an antenna at the back and the aorta is a complex function of geometry. In order to simplify the mathematical representation of the problem, all materials outside the aorta are treated as a single, lossy environment.
As justified in [22], an acceptable material representation of the original geometry is to average the permittivity of the materials $(\mathcal{M})$ based on the ratio of their respective area $\left(A_{m}\right)$ to total area $(A)$,

$$
\bar{\gamma}=\omega \sqrt{\mu \sum_{m \in \mathcal{M}} \frac{A_{m}}{A} \epsilon_{m}},
$$

where $\omega$ is the angular frequency, $\bar{\gamma}$ is the average material propagation constant, $\epsilon_{m}$ is the permittivity of material with indice $m$, and $\mu$ is the permeability of the materials and is assumed to have identical relative permeability of unity. This approach was based on the analogy with a heterogeneous one-dimensional problem with a sequence of material properties, whose accumulated effect, while disregarding transmission and reflection coefficients, may be represented by a homogeneous material with average propagation constant. By averaging permittivities instead of propagation constants, the resulting "average properties" were found to lie within the variation of the different tissues involved and relatively close to the propagation constant average, denoted "true average" in [22].

The channel model is, therefore, constructed as a cylinder of radius $r(\boldsymbol{\theta}[2])$ immersed in a different material and at a distance $R(\theta[1])$ from an antenna in a monostatic radar measurement situation. Both materials are lossy; the cylinder material is "blood" $(\gamma)$ and the surrounding material is the above average material $(\bar{\gamma})$; all material characterizations are originally based on C. Gabriel and S. Gabriel [23].

A third parameter in the model incorporates the fact of subtracting two distinct radar echoes separated by some time interval and during which the aorta radius has changed by $\Delta r(\boldsymbol{\theta}[3])$. Due to the strong attenuation of biological tissue in general, it is expected that the subtraction is necessary to remove clutter from static materials and allow for observing the radar echo from the aorta in presence of much stronger reflections. This subtraction is integrated into the model as it is expected to constitute a common part of any estimation strategy. Furthermore, by expressing the subtraction as a function of actual radial change, the precise temporal behaviour of the aorta radius may be disregarded.

In [24], the theoretic response from a cylinder of arbitrary material in a lossless material with arbitrary propagation speed is developed $\left(\mathbf{C}_{r}\right)$. This expression defines two parameters: $r$, the radius of the cylinder and $R$, the distance at which the response is observed. Here, we have used the far field approximation of this response ( $R$ infinite) thereby assuming that the antenna is sufficiently far from the aortic structure compared with the wavelength in the surrounding material. To account for the phase of the response, $R$ in the factor $e^{j \gamma R}$ has been set to the radius of the cylinder $(r)$. From the edge of the cylinder, the "material" transfer function $\left(\mathbf{M}_{R}\right)$ will account for the phase due to propagation from the antenna to the aorta and back. This assumes a planar propagation approximation between both transmit and receiver antenna and the aorta.

The fact of assuming a far field approximation has two motivations: the expression in [24] assumes an incident plane wave and the distance between the antenna and the 
aorta $(R)$ is close to satisfying the common criterion for the limit of the near-field,

$$
\begin{aligned}
& R_{\text {near field }}=\frac{2 D^{2}}{\lambda}=\frac{2 D^{2} f}{v_{p}} \leq 15 \mathrm{~cm}, \\
& \text { when } f \leq 5 \mathrm{GHz}, v_{p} \geq c_{0} / 5, \\
& R_{\text {near field }} \leq 9 \mathrm{~cm},
\end{aligned}
$$$$
\text { when } f \leq 3 \mathrm{GHz}, v_{p} \geq c_{0} / 5 \text {, }
$$

where $v_{p}$ is the phase velocity of the wave, $D$ is the greater of antenna and target dimension and is chosen as the maximum diameter of the aorta used in this paper. Hence, the wavefront at the aorta is nearly planar, and the reflection likewise back at the antenna. In Figure 2, this model simplification is compared with actual simulation results for $r=10 \mathrm{~mm}$. We observe that the forms of the responses are similar although with a flat factor $F$ separating the two, principally due to the $1 / R^{2}$ round-trip loss factor of cylindrical versus planar propagation. The cylindrical propagation models geometry, sources, and fields that are symmetrical about any appropriately oriented 2D cross-section.

The combined, resulting channel model is hence expressed according to the following equations. The total response $\left(\mathbf{H}_{\boldsymbol{\theta}}\right)$ is first decomposed as the subtraction of independent radar echoes $\left(\mathbf{G}_{r, R}\right)$ corresponding to two distinct radii ( $r$ and $r+\Delta r$ ), which also implies two distinct distances $R$ as this has been defined relative to the front edge of the cylinder,

$$
\begin{aligned}
\mathbf{H}_{\boldsymbol{\theta}}[k] & =\mathbf{G}_{r+\Delta r, R-\Delta r}[k]-\mathbf{G}_{r, R}[k], \\
\mathbf{G}_{r, R}[k] & =\mathbf{M}_{R}[k] \mathbf{C}_{r}[k] .
\end{aligned}
$$

The material transfer function is a simple exponential factor (19), while the cylinder response (20), see Ruck et al. [24], is an infinite series $(T(r, k))$ with complicated terms $\left(A_{n}(r, k)\right)$ in the form of fractions (numerator $N_{n}(r, k)$, denominator $\left.D_{n}(r, k)\right)$ of Bessel functions $\left(J_{n}(x)\right)$ and Hankel functions of the first kind $\left(H_{n}(x)\right)$,

$$
\begin{aligned}
\mathbf{M}_{R}[k] & =\operatorname{Exp}\left\{-j \bar{\gamma}_{k} 2 R\right\}, \\
\mathbf{C}_{r}[k] & =\frac{2 e^{j\left(\bar{\gamma}_{k} r-\pi / 4\right)}}{\sqrt{\bar{\gamma}_{k}}} T(r, k) \\
& =\frac{2 e^{j\left(\bar{\gamma}_{k} r-\pi / 4\right)}}{\sqrt{\bar{\gamma}_{k}}}\left\{\sum_{n=0}^{\infty} A_{n}(r, k)\right\}, \quad \text { where } \\
A_{n}(r, k) & =\left\{\begin{array}{l}
N_{0}(r, k) \\
D_{0}(r, k)
\end{array}-2(-1)^{n} \frac{N_{n}(r, k)}{D_{n}(r, k)}, \quad n \geq 1,\right. \\
N_{n}(r, k) & =\bar{\gamma}_{k} J_{n}\left(\gamma_{k} r\right) J_{n}^{\prime}\left(\bar{\gamma}_{k} r\right)-\gamma_{k} J_{n}^{\prime}\left(\gamma_{k} r\right) J_{n}\left(\bar{\gamma}_{k} r\right), \\
D_{n}(r, k) & =\bar{\gamma}_{k} H_{n}\left(\gamma_{k} r\right) J_{n}^{\prime}\left(\bar{\gamma}_{k} r\right)-\gamma_{k} H_{n}^{\prime}\left(\gamma_{k} r\right) J_{n}\left(\bar{\gamma}_{k} r\right) .
\end{aligned}
$$

3.3. Numerical Evaluation of Lower Bounds. The objective is to evaluate the influence of signal choice upon the FIM (13) and particularly see if a general constraint on center frequency $\left(f_{c}\right)$ and bandwidth $(B)$ emerges. However, in order to evaluate the expression, a definition of the noise process is needed and is assumed to be white:

$$
\mathbf{K}_{n, f}[k, k]=N_{0} \approx \frac{\Gamma_{0}}{\Delta t} .
$$

If not white, and contingent on knowledge of the process, it may be whitened by a suitable transformation which would necessarily imply a transformation of the signal $s$. The present results would then apply to the transformed signal.

The second choice concerns the signal space to search. It is apparent in (13) that only the energy in each frequency bin has an influence on the FIM and is hence invariant to any phase transformation of the signal. For simplicity, the 


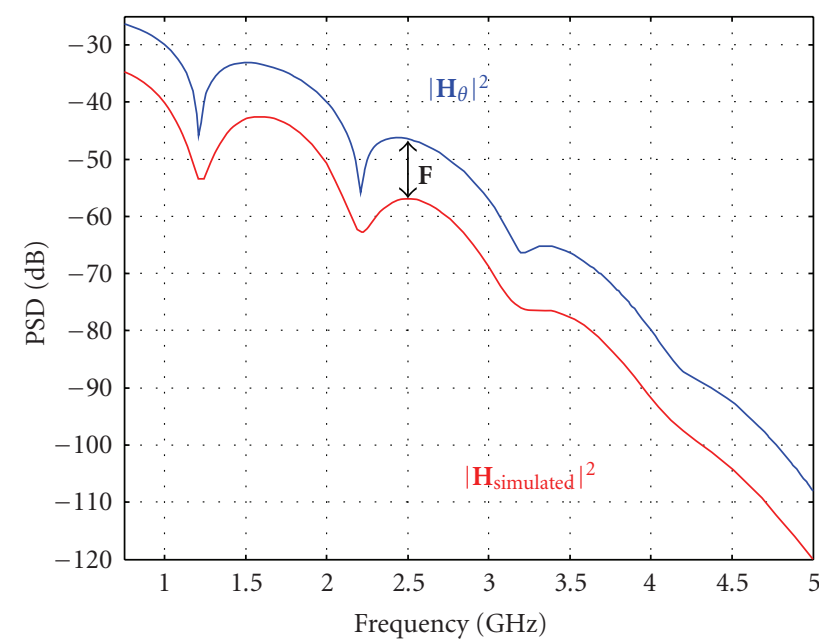

Figure 2: The model $\mathbf{H}_{\boldsymbol{\theta}}$ based on planar propagation $\mathbf{M}_{R}$ combined with the cylinder response $\mathbf{C}_{r}$ compared with simulation results. Using a flat factor of $F=10$, the two PSDs practically overlap, although the model has deeper troughs and more lopsided peaks. The figure is based on results in [22].

energy in each frequency bin over the bandwidth is assumed constant,

$$
\begin{aligned}
|\mathbf{S}[k]|^{2} & =\frac{E_{\mathrm{sd}}}{2 N_{B}}\left\{\operatorname{rect}\left(\frac{k-k_{c}}{N_{B}}\right)+\operatorname{rect}\left(\frac{k+k_{c}}{N_{B}}\right)\right\}, \\
E_{\mathrm{sd}} & \triangleq \sum_{m=0}^{N-1} s[m]^{2}=\sum_{k=-M}^{M}|\mathbf{S}[k]|^{2} \approx \frac{E_{s}}{\Delta t},
\end{aligned}
$$

where $k_{c}, k, N_{B}$ are related to $f_{c}, f, B$; their ratios are all $1 / T$. As in (15), the FIM scales directly as a function of the ratio $E_{s} / \Gamma_{0}$ and we have $E_{s} / \Gamma_{0} \approx E_{s d} / N_{0}$. We have mentioned earlier that this implies that the CRLB may be calculated for a constant ratio and then the value for any other ratio simply scales the CRLB. Therefore, in what follows, this ratio is assumed equal to unity. For clarity, whenever we refer to the CRLB we will assume a ratio of unity, which will result in expected standard deviation on the order of meters whereas the radius is on the order of $10-20 \mathrm{~mm}$ in our application. The assumption is of course that in a practical situation the ratio is sufficiently large for the CRLB to be meaningful (order of $1 \mathrm{~mm}$ ).

In summary, for each value of the CRLB, we are considering the class of signals with equal bandwidth and center frequency and with a signal energy such that $E_{s} / \Gamma_{0}=1$. T is an independent parameter and, therefore, this approach does not constrain the time-bandwidth product, for example, compare a linearly frequency-modulated signal (chirp) to a sinc, each with equal energy.

Finally, relevant ranges on the parameter space must be set. With regards to $\left(f_{c}, B\right)$, and as the FIM is strongly dependent on signal energy at the receiver, it is expected that the CRLB for bands above $5 \mathrm{GHz}$ will be exceedingly high. The calculations will be limited to the intervals

$$
\left(f_{c}, B\right) \in\langle 0.5,5\rangle \mathrm{GHz} \times\langle 0.1,3\rangle \mathrm{GHz} .
$$

With regards to $R$, from infants up to obese adults, it may vary over very large ranges, and will also vary upon position along the aorta for a given individual. Although the variation is not as important, similar remarks apply to $r$. With respect to $\Delta r$, an accurate study over several individuals sets the peak-to-peak radius variations, for normal, adult individuals to $1.09 \pm 0.22 \mathrm{~mm}$ [15]. In a typical measurement setup, the radial variation between two measurements may be any value although limited above by this peak. Relevant, arbitrary ranges have been chosen as

$$
(R, r, \Delta r) \in \Theta=\langle 8,15\rangle \mathrm{cm} \times\langle 8,15\rangle \mathrm{mm} \times\langle 0.05,1\rangle \mathrm{mm} .
$$

In Figures 3(a), 3(b), and 3(c), the results of numerical calculations are displayed. In order to visualize the structure, the values have been truncated to appropriate levels. With respect to Figure 3(c), the average is based on assuming that every element in the parameter space $\Theta$ is equally likely. However, with regard to $\Delta r$, this weighting reflects a less than optimum approach as the performance can be improved if small $\Delta r$ are avoided. It may be possible in a real system to avoid such small values if for each echo the reference is chosen which produces the greatest difference.

\section{Simulations}

In order to verify the expressions and numerical calculations of the lower bound, we have chosen to perform simulations. In principle, verifying a lower bound requires proving that no estimator performs better. If we had found one that did, we would have proven it wrong. On the other hand, showing that an estimator does not violate the lower bound does not constitute a verification unless the estimator was efficient in the statistical sense, or sufficiently close to it. ML estimators are known to be asymptotically efficient, subject to certain conditions, and may therefore qualify. Furthermore, due to the quadric nature of the log-likelihood in the vicinity of $\hat{\boldsymbol{\theta}}_{\mathrm{ML}}$, the Newton-Raphson gradient-based technique is recommended in [25, Chapter 6].

However, the need to calculate the Stochastic Fisher Information Matrix (SFIM), which requires evaluating a set of integrals of functions expressed as infinite series, results in a procedure that proved too slow using available resources. Therefore, a grid-based procedure has been chosen. One consequence is the fact that the grid-based procedure is not efficient unless letting the grid-size tend to zero, which is prohibitive. Therefore, the resulting estimations should not expect to perfectly attain the CRLB, but the bias due to the grid will be chosen sufficiently small to disregard this effect. 
As illustrated schematically in Figure 4, the estimation of $\hat{\boldsymbol{\theta}}_{\mathrm{ML}}$ need not find the closest grid point. It follows that the grid may introduce an additional variance. For multivariate normal (MVN) distributions or if one may assume a point close enough to $\hat{\boldsymbol{\theta}}_{\mathrm{ML}}$, surfaces of equal log-likelihood may be approximated by ellipsoids. If the axes of this ellipsoid is skewed relative to the grid axes, then the point on the grid with lowest log-likelihood may be farther than half a grid step away. The $\log$-likelihood $(l(\boldsymbol{\theta}, \mathbf{x}))$, given an observation, may be expanded in a Taylor series centered at $\hat{\boldsymbol{\theta}}_{\mathrm{ML}}$ [25, Chapter 6],

$$
l(\boldsymbol{\theta}, \mathbf{x}) \approx\left(l\left(\hat{\boldsymbol{\theta}}_{\mathrm{ML}}, \mathbf{x}\right)\right)-\frac{1}{2}\left(\boldsymbol{\theta}-\hat{\boldsymbol{\theta}}_{\mathrm{ML}}\right)^{T} \mathbf{J}\left(\hat{\boldsymbol{\theta}}_{M L}, \mathbf{x}\right)\left(\boldsymbol{\theta}-\hat{\boldsymbol{\theta}}_{\mathrm{ML}}\right),
$$

where $\mathbf{J}\left(\hat{\boldsymbol{\theta}}_{\mathrm{ML}}, \mathbf{x}\right)$ is the SFIM.

The problem inherent in Figure 4 may be avoided by performing an eigenvalue analysis of the SFIM and orienting the axes of the grid along the eigenvectors. Using the FIM instead of the SFIM, this reasoning should still hold on the average, and the expected error introduced by the grid will be bounded by half the grid size in either direction,

By performing an eigenvalue analysis of the SFIM and orienting the axes of the grid along the eigenvectors avoids the problem inherent in Figure 4 by aligning the grid along the ellipsoids. Using the FIM, developed for the CRLB, instead of SFIM, this reasoning should still hold on the average, and hence the error introduced by the grid will be bounded by half the grid size in either direction,

$$
\begin{gathered}
\mathbf{J}\left(\hat{\boldsymbol{\theta}}_{\mathrm{ML}}\right)=\mathbf{E}_{v} \Lambda \mathbf{E}_{v}^{T}, \\
\text { where } \Lambda=\operatorname{diag}\left(\lambda_{1}^{-2}, \lambda_{2}^{-2}, \lambda_{3}^{-2}\right), \\
\left(\boldsymbol{\theta}-\hat{\boldsymbol{\theta}}_{\mathrm{ML}}\right)^{T} \mathbf{J}\left(\hat{\boldsymbol{\theta}}_{\mathrm{ML}}\right)\left(\boldsymbol{\theta}-\hat{\boldsymbol{\theta}}_{\mathrm{ML}}\right)=\mathbf{y}^{T} \Lambda \mathbf{y} \\
\text { where } \mathbf{y}=\mathbf{E}_{v}^{T}\left(\boldsymbol{\theta}-\hat{\boldsymbol{\theta}}_{\mathrm{ML}}\right) .
\end{gathered}
$$

$\mathbf{E}_{v}$ is the matrix with orthonormal eigenvectors arranged in columns and $\Lambda$ contains the eigenvalues along the diagonal. Parameters are real quantities. This means that the error in our estimate of the CRLB follows the classical error introduced by quantization $(q)$ : $\operatorname{VAR}[q]=\Delta^{2} / 12$. Further, by choosing $\Delta$ as a fraction of $\lambda_{i}$, for example, $\Delta=\lambda_{i} / k$, the relative error may be made insignificant,

$$
\sqrt{\lambda_{i}^{2}+\frac{\left(\lambda_{i} / k\right)^{2}}{12}}=\left.\lambda_{i} \sqrt{1+\frac{1}{12 k^{2}}}\right|_{k=3}=1.0046 \lambda_{i}
$$

where $\lambda_{i}$ is the standard deviation along the eigenvector axis $i$.

As for the numerical evaluations of the lower bound, we will select an ideal bandpass signal. As the expressions scale directly with $E_{\mathrm{sd}} / N_{0}$, performing simulations for a single value is sufficient,

$$
\begin{gathered}
E_{\mathrm{sd}}=1.0 \mathrm{~W}=\frac{E_{s}}{\Delta t}, \\
-\frac{10}{B} \leq t \leq \frac{25}{B}, \quad s(t)=\sqrt{2 B E_{s}} \operatorname{sinc}(t B) 2 \pi f_{c} t, \\
S(f)=-j \sqrt{\frac{E_{s}}{2 B}}\left\{\operatorname{rect}\left(\frac{f-f_{c}}{B}\right)-\operatorname{rect}\left(\frac{f+f_{c}}{B}\right)\right\}, \\
\Delta t=\left(20 f_{c}\right)^{-1}, m=0, \ldots, N-1, s[m]=s(m \Delta t) .
\end{gathered}
$$

The estimation of each parameter $\boldsymbol{\theta}_{0} \in \Theta$ is repeated $R=1000$ times with independent instances of the noise random process, which are generated as white, Gaussian random processes,

$$
\{n[m]\} \sim \mathcal{N}\left(\mathbf{0}, \mathbf{K}_{n}\right), \quad \mathbf{K}_{n}=N_{0} \mathbf{I} .
$$

The ratio $E_{\text {sd }} / N_{0}$ is set such that ambiguities are not expected because the expected estimation error becomes much less than the distance between $\boldsymbol{\theta}_{0}$ and the nearest ambiguity,

$$
\frac{E_{\text {sd }}}{N_{0}}=\operatorname{Max}\left\{\frac{\operatorname{CRLB}(\hat{R})}{(0.2 \mathrm{~mm})^{2}}, \frac{\operatorname{CRLB}(\hat{r})}{(0.1 \mathrm{~mm})^{2}}, \frac{\operatorname{CRLB}(\widehat{\Delta r})}{(0.01 \mathrm{~mm})^{2}}\right\} .
$$

This choice assures that the estimation error is above the threshold level in the Ziv-Zakai lower bound. A grid offset from the actual parameter value $\boldsymbol{\theta}_{0}=\left(R_{0}, r_{0}, \Delta r_{0}\right)$ is selected with step size $\lambda_{i} / 3$, extending $4 \lambda_{i}$ in each eigenvector direction,

$$
\begin{aligned}
\tilde{\boldsymbol{\theta}}_{0} & =\boldsymbol{\theta}_{0}+\delta \boldsymbol{\theta}_{0}, \delta \boldsymbol{\theta}_{0} \sim \operatorname{Uniform}\left(\mathbf{0}, \operatorname{diag}\left(\frac{\sigma_{\widehat{R}}}{12}, \frac{\sigma_{\widehat{r}}}{12}, \frac{\sigma_{\widehat{\Delta r}}}{12}\right)\right), \\
\mathbf{y}_{i} & \in\{-12, \ldots, 12\} \cdot \frac{\lambda_{i}}{3}+\mathbf{e}_{v i}^{T} \widetilde{\boldsymbol{\theta}}_{0}, \\
\boldsymbol{\theta} & \in\left\{\mathbf{E}_{v} \mathbf{y}\right\} .
\end{aligned}
$$

Simulation results for various selections of parameters are displayed in Figures 5(a), 5(b), 5(c), 5(d), and 5(e). In Figure 5(a), the CRLB is shown as a function of center frequency; in Figure 5(b), it is displayed as a function of bandwidth, while in Figures 5(c), 5(d), and 5(e) the CRLB is illustrated as functions of $R, r$, and $\Delta r$, respectively.

4.1. Threshold Effect. In the above simulations, the objective was to verify the expressions and numerical calculations of the CRLB. In doing so, the necessity of a sufficient $E_{\mathrm{sd}} / N_{0}$ ratio was emphasized in order to avoid ambiguities, which are not accounted for by the CRLB. In this simulation series, the objective is to illustrate through simulation the point at which the threshold effect becomes visible by successively 


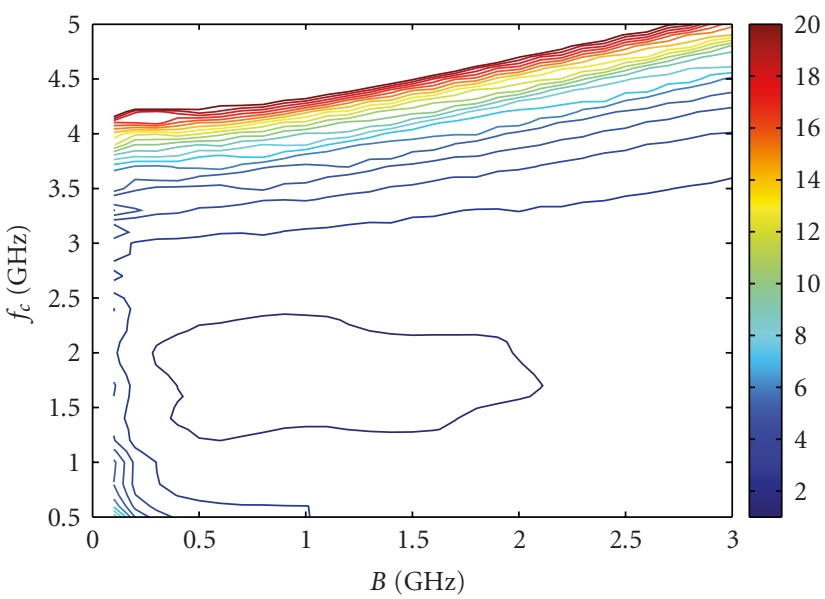

(a) Minimum CRLB

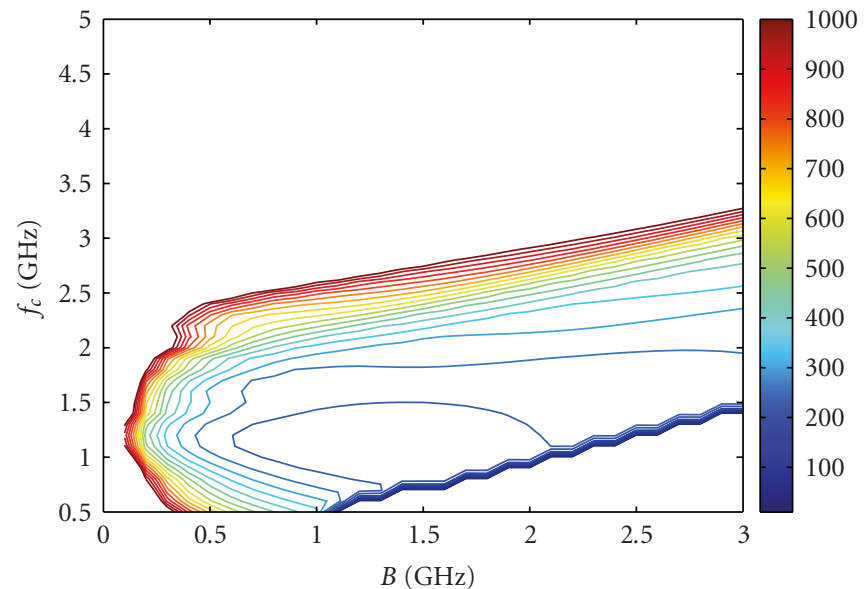

(b) Maximum CRLB

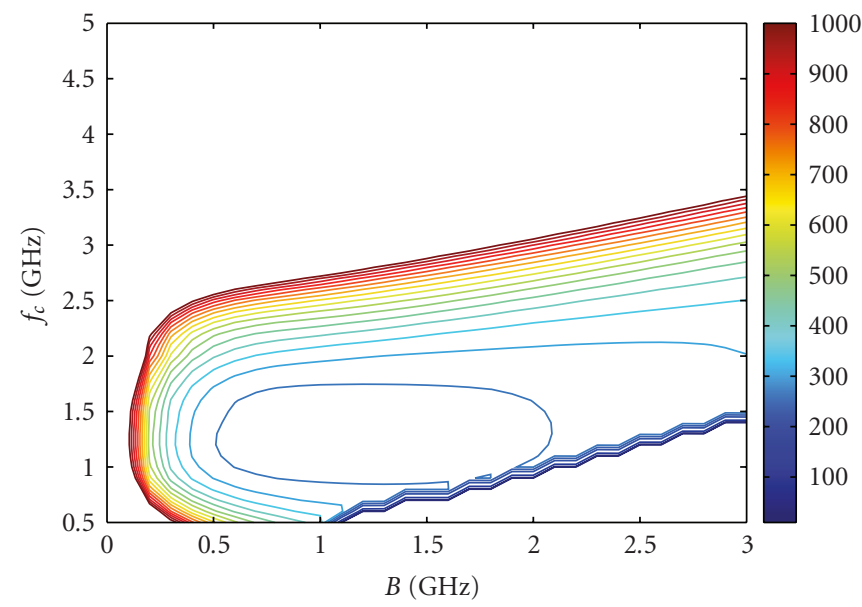

(c) Average CRLB

FIgURE 3: CRLB for $\hat{r}$ over $\Theta$ as a function of $\left(f_{c}, B\right)$.

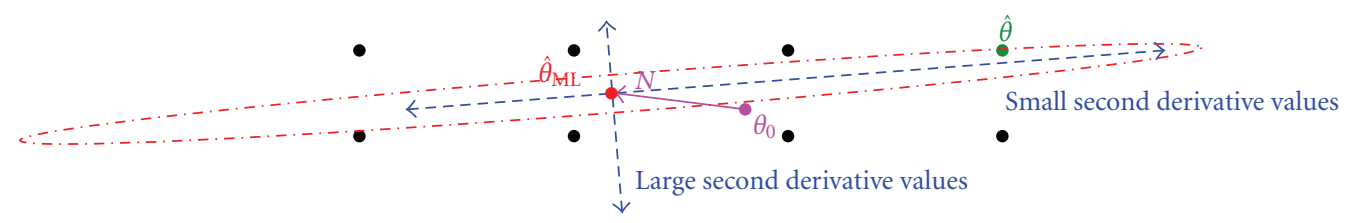

FIgURE 4: Illustrate properties of the grid-based approach to $\hat{\boldsymbol{\theta}}_{\mathrm{ML}}$. Here, $\boldsymbol{\theta}_{0}$ is the actual parameter value, $\hat{\boldsymbol{\theta}}_{\mathrm{ML}}$ is the true minima due to noise $\mathcal{N}$, and $\hat{\boldsymbol{\theta}}$ is the minima on the grid and hence the ML estimate based on the grid-based approach. The figure does not use a rotated grid to adjust to the principle axes of the FIM schematically represented by the dashed-line axes in the figure.

reducing the $E_{s d} / N_{0}$ ratio while all other parameters are kept constant,

$$
\begin{aligned}
\boldsymbol{\theta} & =(13 \mathrm{~cm}, 10 \mathrm{~mm}, 0.6 \mathrm{~mm}) \\
\wedge & \left(f_{c}, B\right)=(1.0 \mathrm{GHz}, 1.0 \mathrm{GHz}), \\
\frac{E_{s d}}{N_{0}} \in\{64.8,69.8,74.8,79.8,82.8,86.8,89.8, & 92.8,94.8,96.8\} \mathrm{dB} .
\end{aligned}
$$

The CRLB in terms of standard deviation for this choice of $\boldsymbol{\theta}$ is $\sigma_{\hat{r}}=7.04 \mathrm{~m}$ at $E_{s d} / N_{0}=0 \mathrm{~dB}$; the observed standard deviations are appropriately scaled $\hat{\sigma}_{\hat{r}} \cdot \sqrt{E_{s d} / N_{0}}$ and compared with the CRLB. The threshold may be defined where the variance becomes larger than the CRLB by a factor of 2 ,

$$
\left.\hat{\sigma}_{\hat{r}} \cdot \sqrt{\frac{E_{\text {sd }}}{N_{0}}}\right|_{\text {threshold }}=\sqrt{2} \sigma_{\hat{r}}=9.96 \mathrm{~m} .
$$


The results of the simulations are illustrated in Figure 5(f), which suggest a threshold around $E_{\mathrm{sd}} / N_{0} \approx 75 \mathrm{~dB}$, which is equivalent to a CRLB of $\sigma_{\widehat{r}} \approx 1.25 \mathrm{~mm}$.

\section{Discussion}

In the previous sections, we have argued for the use of the CRLB in order to describe the performance of radius estimation as a function of the channel parameters. The CRLB for a general transfer function has been derived. We further elaborated a channel model dependent on three parameters: a cylinder of radius $r$ of lossy material is immersed in a lossy material separated from the antenna in a monostatic radar configuration by a distance $R$. The third parameter is the difference in radius $\Delta r$ between two echoes.

In order to verify the CRLBs, simulations have been performed using an estimator which comes sufficiently close to the actual ML estimate value compared with the expected standard deviation of the ML estimate according to the CRLB value. This estimator uses a grid-based approach, oriented according to the eigenvector directions of the FIM in order to improve the performance. The results of these simulations are shown in Figures 5(a) through 5(f). In Figures 5(a) and $5(\mathrm{~b})$, the dependency on system parameters $f_{\mathrm{c}}$ and $B$ is shown. In Figures 5(c), 5(d), and 5(e) the dependency on model parameters $R, r$, and $\Delta r$ is shown, while in Figure 5(f) the threshold effect, where the CRLB is no longer precise, is illustrated.

Given that the results of simulations are realizations of a stochastic variable, confidence intervals have been added to quantify their variations. These confidence intervals are based on the assumption that the distribution of $\hat{\boldsymbol{\theta}}_{\mathrm{ML}}$ may be modeled as a normally distributed random variable. This assumption would naturally be violated if the variance was too large compared with the second derivative of the norm $\left\|\mathcal{X}-\mathbf{Z}_{\boldsymbol{\theta}}\right\|^{2}$ as a function of $\boldsymbol{\theta}$ close to $\boldsymbol{\theta}_{\mathrm{ML}}$. Then the distribution of the estimate of the standard deviation of $\hat{\boldsymbol{\theta}}\left(\sigma_{\widehat{\boldsymbol{\theta}}}\right)$ is a $\chi$-distribution,

$$
\sqrt{\frac{R-1}{\sigma_{\boldsymbol{\theta}}^{2}}} \sigma_{\hat{\boldsymbol{\theta}}}=\sqrt{\sum_{i=1}^{R}\left(\frac{\hat{\boldsymbol{\theta}}_{i}-\mu_{\hat{\boldsymbol{\theta}}}}{\sigma_{\boldsymbol{\theta}}}\right)^{2}} \sim \chi_{R-1} .
$$

Using the distribution of the estimate of $\sigma_{\hat{\theta}}, 99 \%$ confidence intervals can be calculated and are shown in Figures 5(a) through 5(f).

Comparing estimated variance based on simulations to the numerically calculated CRLBs shows that the general dependencies on different parameters correspond very well; all simulation points have a confidence interval that contains the numerically calculated CRLB.

5.1. Interpretations of the CRLB's Dependency on $f_{c}$ and $B$. In Figures 3(a), 3(b), and 3(c), a region of low variance is limited at both low and high center frequencies as well as by the impossible region where the bandwidth is twice or more the center frequency. This low-variance region is also limited at low bandwidths, except when considering the minimum attainable CRLB; at high bandwidths, the variance tends to increase more slowly. In sum, there is a region that seems optimal, and which could loosely be defined by the inequality

$$
\left(\frac{f_{c}-1 \mathrm{GHz}}{0.25 \mathrm{GHz}}\right)^{2}+\left(\frac{B-1.25 \mathrm{GHz}}{0.5 \mathrm{GHz}}\right)^{2} \leq 1
$$

It is true that the minima over the parameter space $\Theta$ does not restrict the use of very narrowband signals, to the contrary, there are values of $\left(f_{c}, B\right)$ which perform very well. However, for such processing to be efficient, it would be necessary to adapt the choice of $\left(f_{c}, B\right)$ to the actual, unknown parameters. Furthermore, the above region appears to perform even better.

As mentioned earlier, the FIM is largest where the average value of the integrand is maximum. This may be used to explain the boundaries of the above region. At low frequency the phase difference between the two radar echoes in the difference (small $\Delta r$ ) is small and results in significant attenuation. At high frequency the tissues are increasingly lossy and the signal is strongly attenuated resulting in higher variance. The fact that the bound for high frequency seems to increase with higher bandwidth simply means that with higher bandwidth a significant lower bandwidth content is included even for higher center frequency.

For low bandwidth, it is clear that beyond some point, the information concerning the radius $r$ of the aorta contained in reflections from the front and rear walls diminishes to the point where only the amplitude of a sinusoid is modulated by the combination of reflections. However, this modulation is coupled with the attenuation due to unknown $R$. Moreover, the channel model in (13) exhibits both notches and peaks due to the resonant behavior of the cylinder, and their locations are hence dependent on $r$. In the best case, the narrow window is centered on a peak. In the worst case, it is centered on a notch which explains the behavior of the worst-case scenario as shown in Figure 3(b) and influences the behavior in Figure 3(c).

Finally, for increasing bandwidth, a greater portion of the spectrum is averaged. When the bandwidth is greater than the effective bandwidth of the radar echo, this average value decreases, hence, increasing estimation variance.

5.2. Implications on $E_{s} / \Gamma_{0}$ for Aorta Radius Estimation. Once $\left(f_{c}, B\right)$ has been chosen and a CRLB at $E_{s} / \Gamma_{0}=1$ has been identified, a required minimum value on $E_{s} / \Gamma_{0}$ is necessary in order to obtain a given accuracy on radius estimation. The first question concerns a relevant value for CRLB.

The values in Figures 3(a), 3(b), and 3(c) all are based on the entire set $\Theta$, however, it is clear that low values of $\Delta r$ have significant impact on received energy and will hence tend to reduce estimation quality. Thus, if measurements may be appropriately arranged to avoid small values of $\Delta r$, then the expected worst-case scenario may improve considerably. On the other hand, $R$ has been limited to $15 \mathrm{~cm}$ in $\Theta$, yet a larger upper limit could be justified. The larger this value, the larger the attenuation in the average material and the less measurements will be precise. In conclusion, the actual parameter range that must be taken into consideration will influence the choice of $E_{s} / \Gamma_{0}$. Conversely, given an upper 


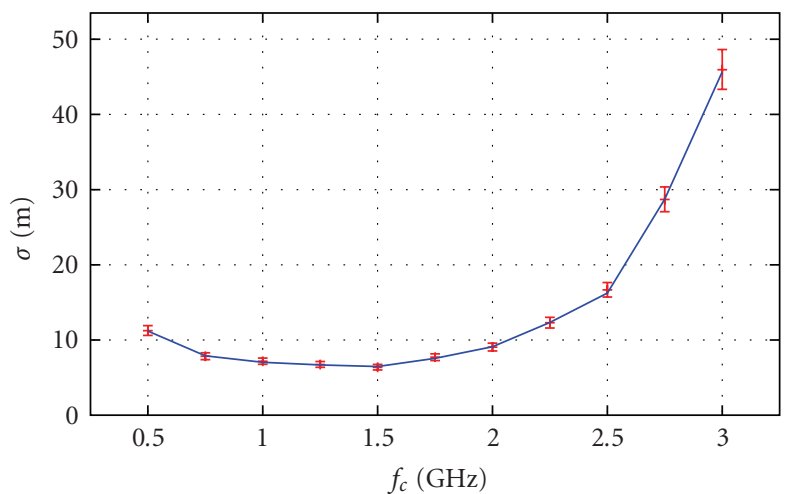

$-\sqrt{\operatorname{CRLB}(\hat{r})}$

$\mapsto \sigma_{\hat{r}} \sqrt{\mathrm{SNR}}$

(a) Variable center frequeny $f_{c}$

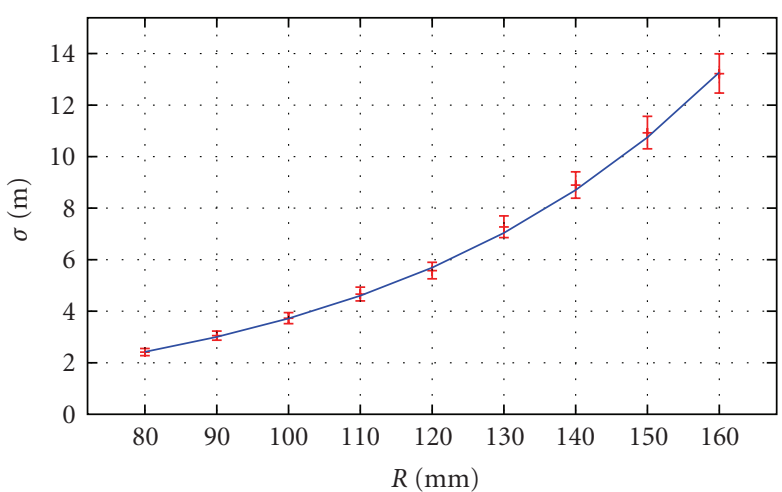

$-\sqrt{\operatorname{CRLB}(\hat{r})}$

$\longmapsto \sigma_{\hat{r}} \sqrt{E_{s} / \Gamma_{0}}$

(c) Variable distance $R$

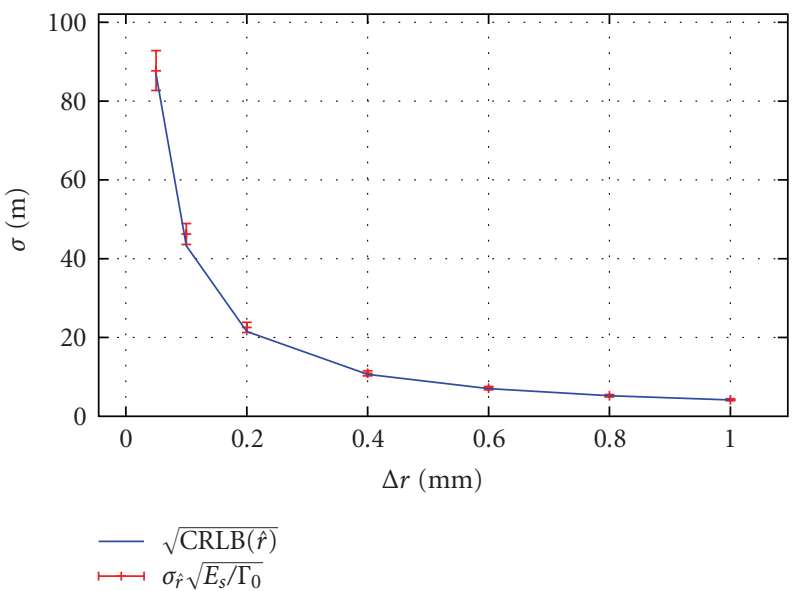

(e) Variable radius difference $\Delta r$

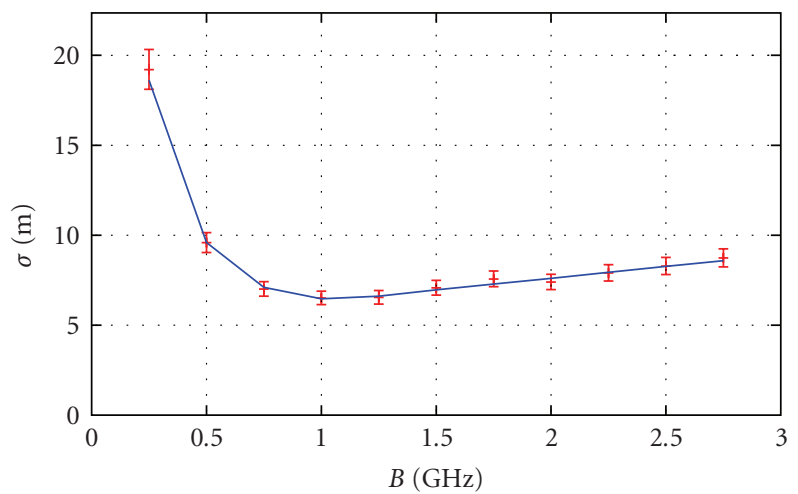

$-\sqrt{\operatorname{CRLB}(\hat{r})}$

$\longmapsto \sigma_{\hat{r}} \sqrt{E_{s} / \Gamma_{0}}$

(b) Variable bandwidth $B\left(f_{c}=1.5 \mathrm{GHz}\right)$

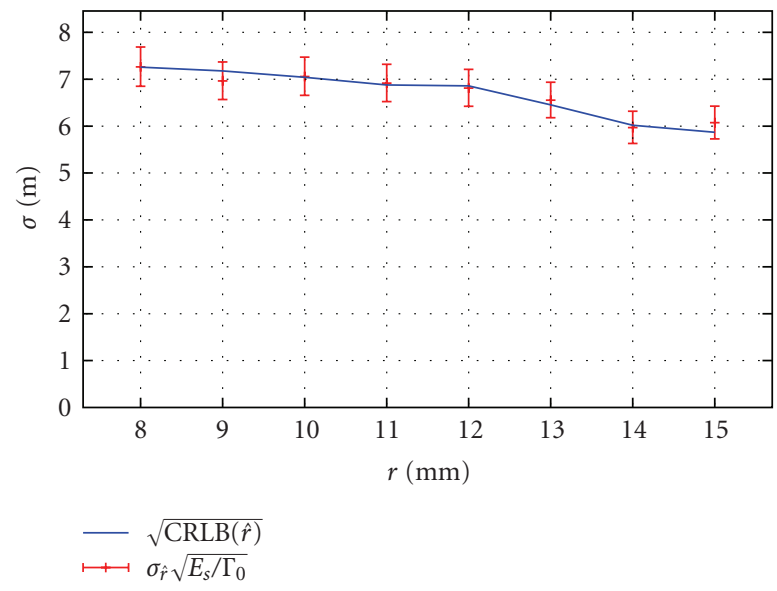

(d) Variable radius $r$

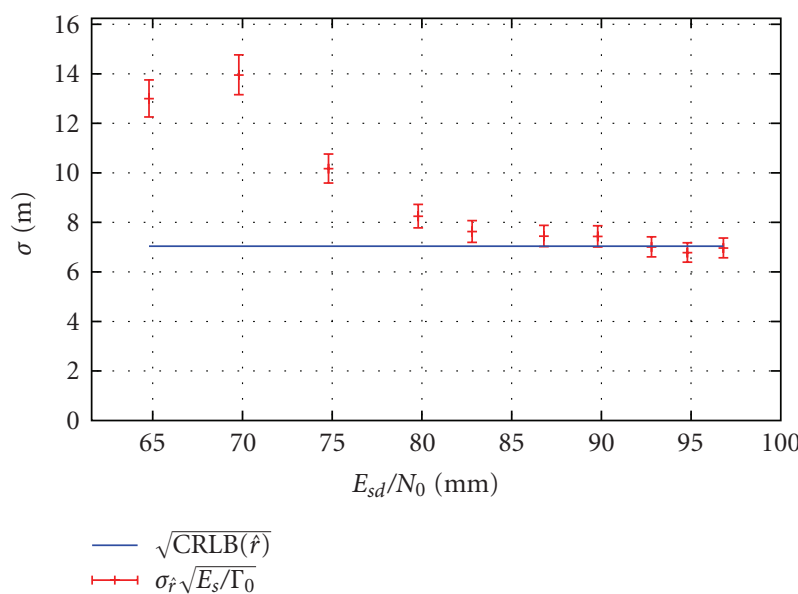

(f) Variable $E_{s d} / N_{0}$ ratio

FigURE 5: CRLB for $\hat{r}$ as a function of a single parameter; default values are $R=13 \mathrm{~cm}, r=10 \mathrm{~mm}, d r=0.6 \mathrm{~mm}, f_{c}=1.0 \mathrm{GHz}$, and $B=1.0 \mathrm{GHz}$. 
bound on $E_{s} / \Gamma_{0}$, a subset of individuals' aorta diameter variations may be measured reliably. With respect to our specific choice for $\Theta$ and taking the worst-case scenario into consideration, a practical value of $\operatorname{CRLB}\left(E_{s} / \Gamma_{0}=1\right)=100 \mathrm{~m}$ may be assumed as a reference.

The required precision depends on the blood-pressure estimation strategy. Let $\sigma_{\hat{r}} \leq 0.1 \mathrm{~mm}$ be assumed adequate and representative. This results in the following requirement on $E_{s} / \Gamma_{0}$ :

$$
\frac{E_{s}}{\Gamma_{0}} \geq\left(\frac{\left.\mathrm{CRLB}\right|_{E_{s} / \Gamma_{0}=1}}{\sigma_{\hat{r}}}\right)^{2}=\left(\frac{100 \mathrm{~m}}{0.1 \mathrm{~mm}}\right)^{2}=10^{12} .
$$

The noise spectral density $\Gamma_{0}$ is bounded below by the thermal noise at the receiver input, $\Gamma_{0} \geq k_{b} T_{\mathrm{abs}}$, where $k_{b}$ is Boltzmann's constant and $T_{\mathrm{abs}}$ is absolute temperature. To quantify the energy $E_{s}$ of the signal, it is necessary to determine the time available for a single observation $(T)$. Whether the signal is pulsed during this period or emits continuously is of no consequence with respect to (13) and (14). Therefore, assume an average power is emitted during $T: P_{s}=E_{s} / T$. The final objective in our project is to estimate blood pressure, which is based on recreating the profile of the Aortic diameter variations, which have a period equal to the heart-beat of an individual. This heart-beat is bounded above by approximately $220 \mathrm{~min}^{-1}=$ 3.7 Hz. Sampling it with frequency $100 \mathrm{~Hz}$, the resulting representation contains 13 harmonics. This implies $T=$ $10 \mathrm{~ms}$ for a single measurement. For $T_{\mathrm{abs}}=300 \mathrm{~K}$ and $B=$ $1 \mathrm{GHz}$,

$$
\begin{aligned}
\frac{E_{s}}{\Gamma_{0}} & =\frac{P_{s} T}{\Gamma_{0}}=\frac{\mathrm{PSD}_{s} T B}{k_{b} T_{\mathrm{abs}}} \geq 10^{12}, \\
P_{s} & \geq \frac{k_{b} T_{\mathrm{abs}} 10^{12}}{T}=0.41 \mu \mathrm{W}, \\
\mathrm{PSD}_{s} & \geq \frac{P_{s}}{B} \\
& =\frac{k_{b} T_{\mathrm{abs}} 10^{12}}{T B} \\
& =4.14 \cdot 10^{-16} \mathrm{~W} / \mathrm{Hz} \\
& =-63.8 \mathrm{dBm} / \mathrm{MHz},
\end{aligned}
$$

where the power spectral density $\left(\mathrm{PSD}_{s}\right)$ is scaled for a onesided spectrum in order to correspond to the thermal noise expression. As a measure of comparison, the FCC has set the general level within the UWB mask to $-41.3 \mathrm{dBm} / \mathrm{MHz}$ [26, Subpart F].

Note that the channel model does not consider the loss in the air-skin reflection and disregards the $1 / R^{2}$ loss due to cylindrical propagation, we have assumed a perfectly $2 \mathrm{D}$ structure, which will add to the above requirement. The airskin reflection loss is on the order of a flat factor $6.4 \mathrm{~dB}$, while the $1 / R^{2}$ loss factor was indicated, for $R \approx 10 \mathrm{~cm}$, to be on the order of $10 \mathrm{~dB}$. Finally, although subtracting two returns will null all static responses, the indirect paths from the aorta will not be suppressed, nevertheless clutter is not considered in the above model. Hence, the above values represent optimistic estimations.

\section{Conclusion}

This article focuses on identifying an optimal signal choice for the estimation of the diameter of a lossy cylinder with slowly-varying radius, in a lossy environment, which has been simplified to that of determining optimal center frequency and bandwidth based on an ideal bandpass filter. The electromagnetic material characteristics are based on tissues as described by C. Gabriel and S. Gabriel [23], and the heterogeneous tissue environment surrounding the aorta has been replaced by a single, average tissue.

By using the Cramér-Rao lower bound (CRLB) as an indicator of estimator performance, it has been shown that both at high and low center frequencies the estimator will perform poorly due to lossy propagation in tissue at high frequencies and the subtraction of signals with small phase shifts at low frequencies. Also at low bandwidths, the estimator will perform less than optimal due to low information content and lack of robustness with respect to parameter values. At high bandwidths, the estimator performs poorly due to indiscriminate filtering. Given our choice of channel model, the optimal selection may be loosely described as an ellipse centered at $1 \mathrm{GHz}$ center frequency and $1.25 \mathrm{GHz}$ bandwidth with axes of $0.5 \mathrm{GHz}$ and $1 \mathrm{GHz}$, respectively.

By considering the necessary signal energy to noise spectral density ratio $\left(E_{s} / N_{0}\right)$ to achieve a radius estimation precision of $0.1 \mathrm{~mm}$, it was found that this ratio should exceed $10^{12}$, when considering the worst case over a range of parameter values. Requiring a measurement every $10 \mathrm{~ms}$, this was found to be equivalent to a mean effect of $0.4 \mu \mathrm{W}$, or equivalently a power spectral density superior to $-63 \mathrm{dBm} / \mathrm{MHz}$, for a bandwidth of $1 \mathrm{GHz}$. These figures do not consider the air-skin interface.

As a future work, we plan to construct a phantom model in which the different parameters are studied in a controlled manner and which may allow for verifying theoretical behavior. Furthermore, using the phantom, the relationship between pressure estimation of pulsatile flow in an elastic tube and diameter as a function of time can be studied in practice.

\section{Acknowledgments}

The voxel model of the human body has been provided by the VOXEL-MAN Group at the University Medical Center Hamburg-Eppendorf, Germany [23]. These data are in turn based on the data set provided by the National Library of Medicine (USA) through the Visual Human Project. The electromagnetic propagation solver, OPEN-TEMSI-FD, has been provided by the Xlim Laboratory, University of Limoges, France [27]. This work is part of the MELODY project, which is funded by the Research Council of Norway under the Contract no. 187857/S10. 


\section{References}

[1] G. Parati, G. Ongaro, G. Bilo et al., "Non-invasive beat-tobeat blood pressure monitoring: new developments," Blood Pressure Monitoring, vol. 8, no. 1, pp. 31-36, 2003.

[2] K. Matthys and P. Verdonck, "Development and modelling of arterial applanation tonometry: a review," Technology and Health Care, vol. 10, no. 1, pp. 65-76, 2002.

[3] J. Y. A. Foo and S. L. Chu, "Pulse transit time as an indirect marker for variations in cardiovascular related reactivity," Technology and Health Care, vol. 14, no. 2, pp. 97-108, 2006.

[4] G. Sharwood-Smith, J. Bruce, and G. Drummond, "Assessment of pulse transit time to indicate cardiovascular changes during obstetric spinal anaesthesia," British Journal of Anaesthesia, vol. 96, no. 1, pp. 100-105, 2006.

[5] A. D. Droitcour, Non-contact measurements of heart and respiration rates with a single-chip microwave doppler radar, Ph.D. dissertation, Department of Electrical Engineering, Stanford University, June 2006.

[6] T. W. McEwan, "Body monitoring and imaging apparatus and method," patent, August 1994.

[7] C. Fowler, J. Entzminger, and J. Corum, "Assessment of ultrawideband (UWB) technology," IEEE Aerospace and Electronic Systems Magazine, vol. 5, no. 11, pp. 45-49, 1990.

[8] I. Immoreev, "Ultra-wideband radars. Features and ways of development," in Proceedings of the 2nd European Radar Conference (EURAD '05), pp. 113-116, October 2005.

[9] X. Li, E. J. Bond, B. D. Van Veen, and S. C. Hagness, "An overview of ultra-wideband microwave imaging via spacetime beamforming for early-stage breast-cancer detection," IEEE Antennas and Propagation Magazine, vol. 47, no. 1, pp. 19-34, 2005.

[10] D. J. Kurrant, E. C. Fear, and D. T. Westwick, "Tumor response estimation in radar-based microwave breast cancer detection," IEEE Transactions on Biomedical Engineering, vol. 55, no. 12, pp. 2801-2811, 2008.

[11] L. Anitori, A. de Jong, and F. Nennie, "FMCW radar for lifesign detection," in IEEE Radar Conference (RADAR '09), pp. 1-6, May 2009.

[12] M. Sugawara, K. Niki, H. Furuhata, S. Ohnishi, and S. Suzuki, "Relationship between the pressure and diameter of the carotid artery in humans," Heart and Vessels, vol. 15, no. 1, pp. 49-51, 2000.

[13] J. Lass, K. Meigas, D. Karai, R. Kattai, J. Kaik, and M. Rossmann, "Continuous blood pressure monitoring during exercise using pulse wave transit time measurement," in Proceedings of the 26th Annual International Conference of the IEEE Engineering in Medicine and Biology Society (EMBC'04), vol. 1, pp. 2239-2242, September 2004.

[14] R. G. Gosling and M. M. Budge, "Terminology for describing the elastic behavior of arteries," Hypertension, vol. 41, no. 6, pp. 1180-1182, 2003.

[15] C. Stefanadis, C. Stratos, C. Vlachopoulos et al., "Pressurediameter relation of the human aorta: a new method of determination by the application of a special ultrasonic dimension catheter," Circulation, vol. 92, no. 8, pp. 2210-2219, 1995.

[16] B. M. Sadler and R. J. Kozick, "A survey of time delay estimation performance bounds," in Proceedings of the 4th IEEE Sensor Array and Multichannel Signal Processing Workshop Proceedings (SAM '06), pp. 282-288, July 2006.

[17] J. P. Ianniello, E. Weinstein, and A. Weiss, "Comparison of the Ziv-Zakai lower bound on time delay estimation with correlator performance," in Proceedings of IEEE International
Conference on Acoustics, Speech and Signal Processing (ICASSP '83), pp. 875-878, April 1983.

[18] P. M. Woodward, Probability and Information Theory, with Applications to Radar, Artech House Books, Boston, Mass, USA, 1980.

[19] H. L. Van Trees, Detection, Estimation and Modulation Theory, Part I, John Wiley \& Sons, New York, NY, USA, 1968.

[20] A. Pommert, K. H. Höhne, B. Pflesser et al., "Creating a highresolution spatial/symbolic model of the inner organs based on the visible human," Medical Image Analysis, vol. 5, no. 3, pp. 221-228, 2001.

[21] National Library of Medicine (NLM), "Visual human project," 1994, http://www.nlm.nih.gov/research/visible/.

[22] L. E. Solberg, I. Balasingham, S.-E. Hamran, and E. Fosse, "A feasibility study on aortic pressure estimation using UWB radar," in Proceedings of IEEE International Conference on Ultra-Wideband (ICUWB '09), pp. 464-468, Vancouver, Canada, September 2009.

[23] C. Gabriel and S. Gabriel, "Compilation of the dielectric properties of body tissues at RF and microwave frequencies," Tech. Rep., Physics Department, King's College, London, UK, June 1996.

[24] G. T. Ruck, D. E. Barrick, W. D. Stuart, and C. K. Krichbaum, Radar Cross Section Handbook, vol. 1, Columbus Laboratories, Battelle Memorial Institute, Plenium Press, Columbus, Ohio, USA, 1970.

[25] L. L. Scharf, Statistical Signal Processing: Detection, Estimation and Time Series Analysis, Addison-Wesley, Reading, Mass, USA, 1991.

[26] FCC, "Title 47 of the code of federal regulations, part 15: Radio frequency devices," February 2002, http://www .access.gpo.gov/nara/cfr/waisidx_02/47cfr15_02.html.

[27] C. Guiffaut, "OPEN-TEMSI-FD: Time Electromagnetic Simulator-Finite Diference," Licence CECILL-C, Laboratory XLIM, University of Limoges, UMR CNRS 6172, http://www.xlim.fr/en/OSA/projets/compat. 